Sanz-Arribas, I. (2018). Influencia de la longitud del vaso sobre las pruebas de socorrismo acuático profesional / The Effect of Pool Length on the Results of Professional Lifeguard Saving Tests. Revista Internacional de Medicina y Ciencias de la Actividad Física y el Deporte vol. 18 (72) pp. 783-795 Http://cdeporte.rediris.es/revista/revista72/artinfluencia983.htm

DOI: http://doi.org/10.15366/rimcafd2018.72.012

\title{
ORIGINAL
}

\section{INFLUENCIA DE LA LONGITUD DEL VASO SOBRE LAS PRUEBAS DE SOCORRISMO ACUÁTICO PROFESIONAL}

\section{THE EFFECT OF POOL LENGTH ON THE RESULTS OF PROFESSIONAL LIFEGUARD SAVING TESTS}

\section{Sanz-Arribas, I.}

Doctor en Ciencias de la Actividad Física y el Deporte. Profesor del Departamento de Educación Física, Deporte y Motricidad Humana. Universidad Autónoma de Madrid (España) ismael.sanz@uam.es

\section{AGRADECIMIENTOS}

Todos los datos que han permitido la elaboración de este estudio, han sido facilitados por la Federación Madrileña de Salvamento y Socorrismo, por lo tanto, en este apartado se agradece la inestimable colaboración de esta entidad y de su equipo docente.

Clasificación UNESCO / UNESCO code: 3212 Salud Pública/ Public Health Clasificación Consejo de Europa/ Council of Europe classification: 17. Otras (Salvamento y socorrismo) / Other (lifesaving)

Recibido 14 de febrero de 2017 Received February 14, 2017 Aceptado 3 de noviembre de 2017 Accepted November 3, 2017

\section{RESUMEN}

En este estudio se ha seleccionado a 2.528 aspirantes a la certificación de socorrista acuático (1.798 hombres y 730 mujeres). Todos ellos han realizado las cuatro pruebas físicas de agua cronometradas que se exigen para trabajar de socorrista en piscinas, instalaciones acuáticas y medio natural en la Comunidad Autónoma de Madrid (España). Del total de los participantes, 1.887 aspirantes realizaron dichas pruebas en vaso de 25 metros y 641 lo hicieron en vaso de 50 metros. El objetivo de este estudio es conocer la influencia de la longitud del vaso en el que se desarrollan estas pruebas físicas, sobre el porcentaje de aprobados y sobre las marcas de tiempo que emplean los participantes para realizarlas. Los resultados de esta investigación demuestran que la longitud del vaso en el que se evalúa a los aspirantes a socorrista, influye significativamente sobre las marcas de tiempo que obtienen y también sobre el número de aprobados. 
PALABRAS CLAVE: Socorrista, longitud del vaso, condición física, evaluación.

\begin{abstract}
In this study 2.528 aspirants for aquatic lifeguard certification (1.798 men and 730 women) were selected. All the participants of the study have performed the four physical tests of chronometric water required in order to work as a lifeguard for swimming pools, aquatic and open water in the Comunidad Autónoma de Madrid (Spain). Of the whole participants, 1.887 aspirants were tested in a 25meter pool and 641 were done so in a 50-meter pool. The aim of this study is to know the influence of the length of the pool in which these physical tests are developed, on the pass rate and on the time needed by the sample to carry them out. The results of this research show that the length of the pool in which the aspirants are evaluated, influences on the time marks obtained by them and also on the number of approved ones.
\end{abstract}

KEY WORDS: Lifeguard, pool length, physical condition, evaluation.

\title{
1. INTRODUCCIÓN
}

La bibliografía establece que los socorristas acuáticos deben conocer y aplicar las medidas de prevención y vigilancia adecuadas al entorno de baño. También deben dominar los métodos y técnicas de intervención del rescate acuático, los primeros auxilios y disponer de un alto nivel de destreza y condición física dentro del agua. Al mismo tiempo se recomienda que estos conocimientos y destrezas se mantengan actualizados a lo largo de toda la vida profesional del socorrista (Ellis \& Associates, 2002; García Sanz et al., 2015; International Life Saving Federation, 2000; International Life Saving Federation, 2007; International Life Saving Federation, 2013; Palacios Aguilar, 2008; Real Federación Española Salvameno y Socorrismo, 2003; Sanz Arribas, 2011; Schwebel et al., 2010; The United States Lifesaving Association, 2016). Por lo tanto, la adquisición y dominio de estas habilidades y destrezas, es un objetivo prioritario para la formación del socorrista acuático. Aunque todos estos parámetros influyen de forma considerable sobre el nivel de competencia del socorrista, este estudio se centra en las pruebas físicas cronometradas que se utilizan para la evaluación del nivel de destreza del socorrista dentro del agua. En relación con esta cuestión, es importante destacar que existen muchas entidades u organismos públicos y privados, que establecen o recomiendan objetivos y criterios de evaluación diferentes para la obtención de un mismo certificado o equivalente (Consejería de Sanidad y Consumo, 2006; Cruz Roja Española, 2017; Federación Madrileña de Salvamento y Socorrismo, 2017; International Life Saving Federation, 2000; International Life Saving Federation, 2013; Ministerio de Trabajo e Inmigración, 2011; The United States Lifesaving Association, 2016). Precisamente la falta de armonización existente en los objetivos y criterios de evaluación de los cursos de formación de socorrista acuático, podría provocar diferencias importantes en el nivel de competencia de los profesionales que trabajan en entornos similares. 
Al margen de esta importante cuestión, también sucede que algunos de los criterios de evaluación recomendados o establecidos para examinar a los socorristas acuáticos, no se protocolizan adecuadamente, o lo hacen de tal forma, que existe la posibilidad de que los aspirantes a socorrista, puedan ser evaluados en condiciones diferentes al realizar un mismo test. Un ejemplo de esto que se está señalando, es la ausencia generalizada de tablas de referencia que establezcan la influencia de la longitud del vaso, sobre las marcas de tiempo exigidas a los aspirantes a socorrista en las pruebas físicas de piscina. De hecho, se sabe que las marcas de tiempo obtenidas en las pruebas de natación disputadas en vasos de 25 metros, son significativamente mejores que las que se obtienen en vasos de 50 metros (Keskinen et al., 2007; Koch-Ziegenbein et al., 2013; Wolfrum et al., 2013; Wolfrum et al., 2014). Esto se debe a que las pruebas desarrolladas en vaso de 25 metros, permiten que el deportista realice más virajes y por lo tanto, pueda empujarse más veces en la pared al recorrer una distancia establecida. Disponer de un mayor número de impulsos sobre la pared, permite que el nadador se desplace más rápido y con menor gasto energético durante la fase de deslizamiento posterior al viraje. En esta misma línea, algunos autores han determinado que nadar una misma distancia en vaso de 25 metros, provoca una frecuencia cardíaca máxima y una acumulación de ácido láctico en sangre, inferiores a las que se producen en las pruebas disputadas en vasos de 50 metros (Keskinen et al., 2007; Lowensteyn et al., 1994). Por estas razones, algunas entidades deportivas aplican tablas de conversión que permiten anular o atenuar, la influencia de la longitud del vaso sobre las marcas de tiempo que acreditan los deportistas en las diferentes pruebas. Así, las marcas mínimas de tiempo que exigen estas instituciones deportivas a sus competidores para poder participar en determinados eventos, dependen del tipo de vaso en el que se han sido acreditadas previamente (Real Federación Española Natación, 2016; Real Federación Española Salvamento Socorrismo, 2016). No obstante, estas tablas de conversión están pensadas y diseñadas para ser aplicadas en las competiciones deportivas de natación y/o salvamento acuático deportivo y por lo tanto, estos mismos baremos no deberían aplicarse directamente a las marcas de tiempo exigidas a los aspirantes a socorrista acuático, ya que las pruebas, la población que las realiza y su finalidad, son completamente distintas. Por otro lado, hay instituciones que establecen los límites de tiempo de las pruebas cronometradas de agua, para evaluar a los aspirantes a socorrista en vasos de 25 metros, incluso hay instituciones que también exigen la realización de pruebas físicas en aguas abiertas (Consejería de Sanidad y Consumo, 2006; International Life Saving Federation, 2007; Ministerio de Trabajo e Inmigración, 2011). Sin embargo, no parece un acierto prescindir de los vasos de 50 metros para desarrollar cursos de formación de socorrista, porque estas instalaciones son claramente válidas para este fin y porque además, actualmente hay una gran disponibilidad de ellas. Es más, la utilización de vasos de 50 metros parece especialmente adecuada para formar a socorristas que aspiran a trabajar en aguas abiertas o espacios acuáticos naturales.

Por todo ello, el objetivo de este estudio es conocer la influencia de la longitud del vaso en el que se desarrollan los test que exige la Orden de la Comunidad de Madrid (Consejería de Sanidad y Consumo, 2006), sobre las marcas de tiempo que obtienen los aspirantes a socorrista acuático. Al mismo 
tiempo, se pretende conocer si esta variable influye sobre el número de aspirantes que aprueba o suspende dichas pruebas. La consecución de estos objetivos permitiría replantear los límites de tiempo exigidos a los socorristas, dependiendo de la longitud del vaso en el que se desarrolla la evaluación. De ese modo, los aspirantes a socorrista serían examinados en igualdad de condiciones, con independencia de la longitud del vaso en el que se desarrollan las pruebas.

Por último, se señala que este artículo forma parte de una amplia investigación vinculada al nivel de condición física y destreza en el agua de los socorristas.

\section{METODOLOGÍA}

En relación a las normas éticas y requisitos para la participación en el estudio, se indica que todos los participantes del estudio son mayores de 16 años y han superado el $4^{\circ}$ curso de la Enseñanza Secundaria Obligatoria (E.S.O.) o equivalente. Además, han presentado un certificado médico oficial, en el que consta que no padecen alguna enfermedad infecto-contagiosa y que son aptos para realizar ejercicio físico en el agua. En lo referente a las normas éticas, se ha mantenido el anonimato de todas las personas que han formado parte del estudio. Para la realización de este estudio, no se ha utilizado ningún método invasivo o lesivo que pudiera afectar a los participantes. También se señala que se han respetado todas las normas éticas de investigación y de protección de datos, tanto nacionales como internacionales. En resumen, en ningún caso se han invadido los límites que la privacidad y el respeto que las personas exigen.

El estudio está realizado con 2.528 aspirantes al certificado de socorrista acuático (1.798 hombres $71,1 \%$ y 730 mujeres $28,8 \%$ ). Todos los participantes del estudio recibieron la formación necesaria para la obtención de dicho certificado entre los años 2006 y 2016. Todos ellos fueron cronometrados al realizar las cuatro pruebas físicas acuáticas que exige la Comunidad de Madrid (Consejería de Sanidad y Consumo, 2006), para poder ejercer de socorrista en piscinas, instalaciones acuáticas y medio natural en dicho territorio. Del total de participantes del estudio, $1.887(74,6 \%)$ aspirantes realizaron dichas pruebas en vaso de 25 metros y los 641 (25,4\%) restantes, lo hicieron en vaso de 50 metros. En relación a la distribución de los sexos en los diferentes vasos, se indica que el $74,5 \%$ de los hombres (1.341) y el $74,7 \%$ de las mujeres (546), realizaron las pruebas en vaso de 25 metros, mientras que $25,4 \%$ de los hombres (457) y el $25,2 \%$ de las mujeres (184), realizaron las pruebas en vaso de 50 metros. Para conocer la influencia de la longitud del vaso, sobre las marcas de tiempo obtenidas en las pruebas físicas cronometradas, se han comparado las marcas de tiempo logradas por los aspirantes que superaron todas las pruebas en vaso de 25 metros, con las marcas de tiempo de todos aquellos que superaron estas mismas pruebas en vaso de 50 metros. Aquellos aspirantes que no superaron alguna de las pruebas exigidas (Consejería de Sanidad y Consumo, 2006), fueron incluidos en el grupo de no aptos y por lo tanto, sólo fueron considerados para conocer el porcentaje de aprobados y suspensos. Por otro lado, se ha decidido no valorar la influencia del sexo sobre los resultados de las pruebas físicas, porque se considera que las personas que hacen uso de los espacios 
acuáticos vigilados por socorristas, sólo están interesadas en conocer que el o la socorrista es competente para ocupar el puesto de trabajo y no en su sexo.

A continuación se realiza una breve descripción de las pruebas físicas y sus límites de tiempo:

I. Prueba 300 metros de nado crol o braza: El aspirante a socorrista debe nadar ininterrumpidamente durante 300 metros a estilo crol y/o braza. El tiempo límite de esta prueba es: 8 minutos.

II. Prueba combinada I: Entrada al agua con avistamiento de la víctima con material auxiliar de rescate adecuado a la víctima. La víctima debe simular que se encuentra en situación de bajo nivel de estrés acuático. Nado de 50 metros de aproximación, toma de contacto con la víctima y traslado durante 50 metros con técnica correcta (con valoración continua de la víctima). El tiempo límite de esta prueba es: 3 minutos 30 segundos. Una vez terminada la parte cronometrada de la prueba, el aspirante debe extraer a la víctima del agua, realizar la valoración y las maniobras de reanimación cardio-pulmonar básica (R.C.P.) durante 3 minutos en un maniquí de R.C.P. Tal y como se puede leer en la descripción de la prueba, el material que debe emplearse en el rescate no está definido, por lo tanto, el examinador puede indicar al aspirante que realice la parte cronometrada de esta prueba, con cualquiera de los materiales de rescate individual habituales (aro salvavidas, tubo de rescate, boya torpedo...). En esta línea, estudios precedentes han concluido que el tubo de rescate y el aro salvavidas, son igualmente rápidos en rescates de 50 y 100 metros, con víctimas inconscientes y en aguas tranquilas (Sanz Arribas, 2015; Sanz Arribas, 2016), aunque también hay estudios que difieren en esto (Barcala-Furelos et al., 2016). Al margen de esta discrepancia en la bibliografía, se indica que los resultados de esta prueba no serán utilizados para conocer la influencia de la longitud del vaso sobre las marcas de tiempo, porque se considera que tanto las características físicas de las sujetos que simularon ser víctimas (peso, complexión, sexo, talla), como su comportamiento en el momento de ser rescatadas (consciente, inconsciente), pueden influir de forma considerable sobre los tiempos acreditados por los aspirantes. Es decir, la prueba no está normalizada. No obstante, al igual que sucede con el resto de pruebas, todos los participantes del estudio que no superaron este test, fueron incluidos en el grupo de no aptos.

III. Prueba combinada II: Entrada al agua con avistamiento de la víctima. Nado de 50 metros de aproximación y 15 metros de buceo ininterrumpido hasta la recogida de una víctima (maniquí) que se encuentra en el fondo del vaso. Por último, el aspirante debe realizar 35 metros de traslado del maniquí, sin que las vías respiratorias de este se vean obstaculizadas por el agua o por las manos del aspirante. El tiempo límite de esta prueba es de 3 minutos. Se destaca que en esta prueba siempre se utiliza un maniquí normalizado, lleno de agua y estanco, que será descrito en el apartado de recursos materiales. En consecuencia, todos los participantes del estudio han realizado la prueba combinada II con el mismo tipo de maniquí. Es decir, la víctima está estandarizada y es inerte. Por otro lado, la normativa de Comunidad de Madrid establece que la profundidad mínima a la que se debe encontrar el 
maniquí durante la prueba, es de 180 centímetros (Consejería de Sanidad y Consumo, 2006). Dicho esto, las instalaciones en las que se han desarrollado los cursos que han permitido obtener los resultados de este estudio, cumplían con este requisito.

En lo que respecta a las pruebas físicas, según la normativa referenciada (Consejería de Sanidad y Consumo, 2006), para poder ejercer de socorrista en piscinas, instalaciones acuáticas de la Comunidad de Madrid, es necesario que el aspirante supere las anteriores tres pruebas. No obstante, si el aspirante quiere obtener la certificación para poder trabajar de socorrista en piscinas, instalaciones acuáticas y medio natural de la Comunidad Autónoma de Madrid, es necesario superar un cuarto test que requiere el uso de aletas y que no es demandado para poder trabajar en piscinas. Este criterio tiene bastante sentido, ya que las distancias y características de los rescates que se demandan en los espacios naturales, hacen totalmente aconsejables la utilización de las aletas (Palacios-Aguilar et al., 2012; Palacios Aguilar, 2012; Sanz Arribas et al., 2017).

IV. Prueba 100 metros rescate con aletas: De pie en el borde de la piscina o en la orilla del medio natural, con las aletas en la mano, entrada dentro del agua. Realizar nado de salvamento durante 50 metros, recoger a una víctima (maniquí) inconsciente situada en el fondo. Remolcarla otros 50 metros sin que el agua pase por encima de la cabeza de la víctima. Extracción de la víctima. Todo ello realizado en un tiempo máximo de dos minutos y treinta segundos. En esta prueba se utiliza el mismo maniquí normalizado que en la prueba combinada II, por lo tanto, la víctima está estandarizada y es inerte. Además, todos los participantes realizaron las pruebas en piscinas. Por otro lado, hay estudios que demuestran que los diferentes tipos de aletas, influyen sobre el tiempo necesario para realizar un rescate. En concreto, se afirma que las aletas de fibra y las aletas más largas, aportan mayor velocidad al rescate que las de goma, especialmente si las aletas son muy cortas (Abraldes et al., 2010; Palacios Aguilar, 2010). Las aletas utilizadas en este estudio no han sido siempre las mismas, pero todas ellas se correspondían con el tipo de aleta que habitualmente se emplean en el entrenamiento de la natación, es decir, las aletas eran de goma y de longitud y rigidez media. En ningún caso se emplearon aletas largas o de fibra. Por todo ello, los resultados de esta prueba deben interpretarse con prudencia, ya que podrían verse influidos por el tipo de aleta empleado por los aspirantes. Aun así y teniendo en cuenta que las características de las aletas empleadas en este trabajo son muy similares, se ha considerado que merece la pena mostrar los resultados de esta prueba.

Además de los matices especificados en la descripción de las cuatro pruebas utilizadas en esta investigación, a continuación se enumeran las causas por las que los aspirantes eran calificados con apto, o no apto en las pruebas:

- Superar el límite de tiempo establecido para cada prueba.

- Agarrarse al bordillo o a la corchera durante la ejecución de la prueba. 
- En aquellas pruebas en las que es necesario remolcar el maniquí, el agua o las manos del aspirante no deben cubrir las vías respiratorias del maniquí.

- Criterio técnico del examinador. Se aclara que el docente puede llegar a considerar que el aspirante no posee las competencias o el nivel de destreza necesarios para rescatar a otra persona en el agua, sin comprometer su propia seguridad o la de la víctima, incluso cuando el aspirante supera los criterios cuantitativos.

\subsection{RECURSOS MATERIALES}

Los recursos materiales necesarios para realizar este estudio han sido los siguientes:

- Vaso de 50 metros de largo y 180 centímetros de profundidad mínima en el lugar de colocación del maniquí.

- Vaso de 25 metros de largo y 180 centímetros de profundidad mínima en el lugar de colocación del maniquí.

- En la prueba 100 metros combinada Il y en la prueba 100 metros rescate con aletas, se utilizaron maniquíes de salvamento y socorrismo acuático homologados por la Real Federación Española de Salvamento y Socorrismo. Las pruebas se realizan con maniquíes estancos y llenos de agua.

- En la prueba combinada I, se utilizaron aros salvavidas homologados para ser utilizados en las instalaciones acuáticas de la Comunidad Autónoma de Madrid y/o tubos de rescate, homologados por la Federación Española de Salvamento y Socorrismo para poder ser utilizados en las competiciones deportivas.

- Cronómetros modelo Casio HS-30W, resistentes al agua y con memoria para 10 parciales.

- Aletas de goma de longitud media. Este tipo de aletas se corresponde con el que habitualmente se emplea en la natación y su entrenamiento.

- Silbatos para dar la señal de comienzo de la prueba cronometrada.

- Todos los participantes del estudio realizaron las pruebas sin gafas de natación.

\subsection{RECURSOS HUMANOS}

- Equipo docente de la Federación Madrileña de Salvamento y Socorrismo, acreditado por la C.S.C. de la C.A.M. para poder impartir este tipo de formación (Consejería de Sanidad y Consumo, 2006). 
- Alumnos que se prestaron voluntarios para asumir el rol de víctimas en la prueba combinada I.

\section{RESULTADOS}

Para el análisis estadístico se ha utilizado el programa "IBM SPSS Statistics 19".

\subsection{DESCRIPTIVOS}

De los 2.528 aspirantes a socorrista que formaron parte de esta investigación, 2.288 (90,5\%) superaron las cuatro pruebas evaluadas en este estudio, mientras que $240(9,5 \%)$ suspendieron al menos una de las cuatro pruebas. El número y porcentaje de aspirantes aptos y no aptos que se produjo en los diferentes vasos, se muestran las siguientes figuras:

1887 aspirantes en vaso de 25 metros

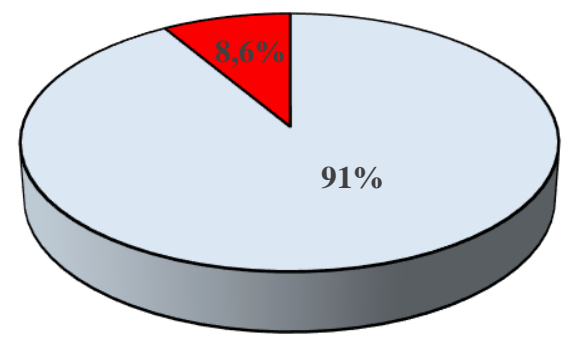

DAptos (1724) QNo aptos (163)

Figura 1. Porcentaje de aptos y no aptos en vaso de 25 metros.

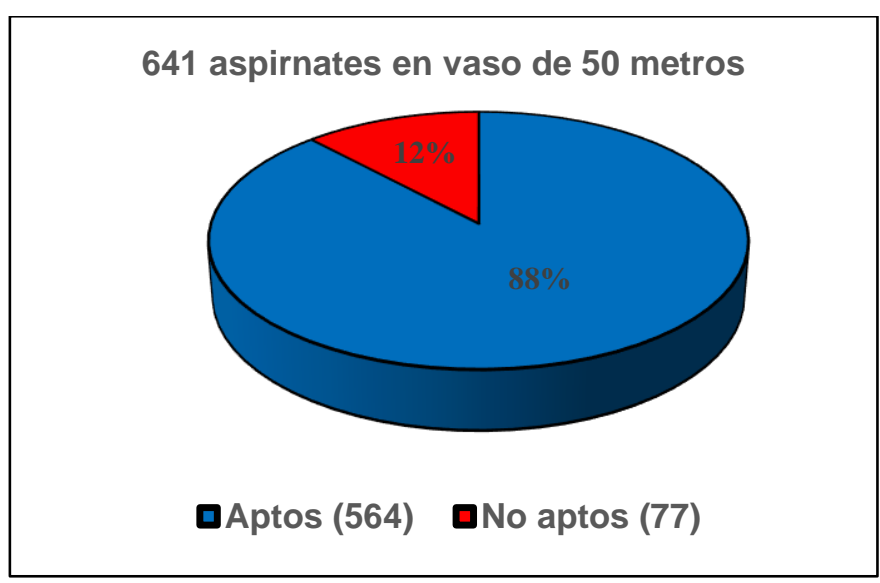

Figura 2. Porcentaje de aptos y no aptos en vaso de 50 metros 
Rev.int.med.cienc.act.fís.deporte - vol. 18 - número 72 - ISSN: 1577-0354

Tabla 1. Estadísticos descriptivos de las pruebas evaluadas en esta investigación

\begin{tabular}{|c|c|c|c|c|}
\hline \multicolumn{5}{|c|}{ Estadísticos descriptivos } \\
\hline Pruebas & $\begin{array}{c}\text { Tiempo en } \\
\text { 300 nado libre }\end{array}$ & $\begin{array}{c}\text { Tiempo en } \\
\text { combinada I** }\end{array}$ & $\begin{array}{c}\text { Tiempo en } \\
\text { combinada II }\end{array}$ & $\begin{array}{c}\text { Tiempo en } \\
\text { 100 aletas }\end{array}$ \\
\hline $\begin{array}{c}\text { Total } \\
\text { participantes }\end{array}$ & $2.528(100 \%)$ & $2.528(100 \%)$ & $2.528(100 \%)$ & $2.528(100 \%)$ \\
\hline No aptos & $93(3,7 \%)$ & $79(3,1)$ & $230(9,1 \%)$ & $69(2,7 \%)$ \\
\hline Media $^{*}$ & 366,6 & 163,7 & 149,9 & 136,0 \\
\hline Desv. típ. $^{*}$ & 62,2 & 20,6 & 19,4 & 23, \\
\hline
\end{tabular}

${ }^{*}$ Tiempos expresados en segundos.

** Puesto que el test combinada I no está estandarizada, sólo se mostrarán los estadísticos descriptivos, pero no se analizará con la prueba t.

Tabla 2. Estadísticos descriptivos por prueba

\begin{tabular}{|c|c|c|c|c|c|c|}
\hline \multicolumn{7}{|c|}{ Estadísticos de grupo } \\
\hline & Tamaño de piscina & $\mathrm{N}$ & $\begin{array}{l}\text { Media en } \\
\text { segundos }\end{array}$ & $\begin{array}{l}\text { Diferencia de } \\
\text { medias en } \%\end{array}$ & $\begin{array}{l}\text { Desviación típ. } \\
\text { En segundos }\end{array}$ & $\begin{array}{c}\text { Error típ. de la } \\
\text { media }\end{array}$ \\
\hline \multirow[t]{2}{*}{ Tiempo en 300 nado libre } & Corta (25 metros) & 1.724 & 360,6 & \multirow[t]{2}{*}{$6,2 \%$} & 61,6 & 1,4 \\
\hline & Larga (50 metros) & 564 & 384,8 & & 60,4 & 2,5 \\
\hline \multirow[t]{2}{*}{ Tiempo en combinada II } & Corta (25 metros) & 1.724 & 148,8 & \multirow[t]{2}{*}{$2,8 \%$} & 19,4 & ,4 \\
\hline & Larga (50 metros) & 564 & 153,1 & & 19,2 & ,8 \\
\hline \multirow[t]{2}{*}{ Tiempo en 100 aletas } & Corta (25 metros) & 1.724 & 135,3 & \multirow[t]{2}{*}{$1,9 \%$} & 22,7 &, 5 \\
\hline & Larga (50 metros) & 564 & 138,0 & & 23,9 & 1 \\
\hline
\end{tabular}

Tabla 3. Pruebas t para comparación de los tiempos de las pruebas y las marcas de tiempo obtenidas en diferentes vasos.

\begin{tabular}{|c|c|c|c|c|c|c|c|c|c|}
\hline \multicolumn{10}{|c|}{ Prueba de muestras independientes } \\
\hline & \multicolumn{2}{|c|}{$\begin{array}{l}\text { Prueba de } \\
\text { Levene para la } \\
\text { igualdad de } \\
\text { varianzas }\end{array}$} & \multicolumn{7}{|c|}{ Prueba t para la igualdad de medias } \\
\hline & \multirow[b]{2}{*}{$\mathrm{F}$} & \multirow[b]{2}{*}{ Sig. } & \multirow[b]{2}{*}{$\mathrm{t}$} & \multirow[b]{2}{*}{ gl } & \multirow{2}{*}{$\begin{array}{l}\text { Sig. } \\
\text { (bilater } \\
\text { al) }\end{array}$} & \multirow{2}{*}{$\begin{array}{c}\text { Difere } \\
\text { ncia } \\
\text { de } \\
\text { media } \\
\text { s }\end{array}$} & \multirow{2}{*}{$\begin{array}{l}\text { Error típ. } \\
\text { de la } \\
\text { diferenci } \\
\text { a }\end{array}$} & \multicolumn{2}{|c|}{$\begin{array}{c}95 \% \text { Intervalo de } \\
\text { confianza para la } \\
\text { diferencia }\end{array}$} \\
\hline & & & & & & & & Inferior & Superior \\
\hline $\begin{array}{l}\text { Tiempo en } 300 \text { metros } \\
\text { nado libre }\end{array}$ & 0,101 & .750 & $-8,142$ & 2.287 & 000 & $-24,2$ & 2,9 & -30 & $-18,3$ \\
\hline $\begin{array}{c}\text { Tiempo en } 100 \text { metros } \\
\text { combinada II }\end{array}$ & ,182 & .670 & $-4,611$ & 2.287 & 000 & $-4,3$ & ,9 & $-2,4$ & $-6,1$ \\
\hline $\begin{array}{c}\text { Tiempo en } 100 \text { metros } \\
\text { aletas }\end{array}$ & 2,380 & .123 & $-2,356$ & 2.287 & 019 & $-2,6$ & 1,1 & $-4,8$ &,- 4 \\
\hline
\end{tabular}

\section{DISCUSIÓN}

Una vez presentados los resultados de este estudio, se puede observar que la longitud del vaso en el que se desarrollan las pruebas físicas 
cronometradas de agua, condiciona el porcentaje de aspirantes que suspende alguna de las pruebas físicas exigidas por la Comunidad de Madrid. De hecho, el $8.6 \%$ de los aspirantes a socorrista que realizaron los cuatro test en vaso de 25 metros, no superó alguna de las pruebas exigidas. En cambio, cuando estas mismas pruebas se desarrollaron en vasos de 50 metros, el porcentaje de aspirantes a socorrista que suspendió una o más de esas mismas pruebas, ascendió al $12 \%$. También se puede afirmar que la longitud del vaso en el que se desarrollan estos test, influye de forma significativa sobre las marcas obtenidas por los aspirantes que superaron todas las pruebas. En concreto, hay diferencias estadísticamente significativas en las tres pruebas analizadas en esta investigación: $(p \leq 0,001)$ en la prueba 300 metros crol y/o braza, $(p \leq 0,001)$ en la prueba combinada II y $(\mathrm{p}=0,019)$ en la prueba 100 metros rescate con aletas. Estos resultados coinciden con las conclusiones de las investigaciones realizadas en el ámbito de la natación de competición (Keskinen et al., 2007; Koch-Ziegenbein et al., 2013; Lowensteyn et al., 1994; Wolfrum et al., 2013; Wolfrum et al., 2014). En este sentido, resulta paradójico que las instituciones deportivas que organizan sus pruebas acuáticas en vasos de diferentes tamaños, utilicen tablas de conversión para limitar la influencia de la longitud del vaso sobre los resultados deportivos (Real Federación Española Natación, 2016; Real Federación Española Salvamento Socorrismo, 2016) y en cambio, no se han encontrado tablas de conversión que desempeñen esta misma función en el ámbito de la evaluación de los socorristas. Por otro lado, no parece un acierto utilizar las mismas tablas que se emplean en el ámbito deportivo para evaluar también a los socorristas. Esto se debe a que el nivel de destreza (velocidad de nado, técnica y condición física...) de los deportistas acuáticos, no es, ni es necesario que sea el mismo que el de las personas que aspiran a ser socorristas. Además hay que añadir, que las pruebas utilizadas para evaluar a los socorristas, son diferentes y no tienen la misma finalidad que las pruebas de competición deportiva. Con independencia de lo anterior, no hay que olvidar que recorrer 300 metros en un vaso de 25 metros, permite tocar y empujarse sobre la pared 6 veces más, que recorrer esa misma distancia en un vaso de 50 metros. En el caso de las otras dos pruebas analizadas en este trabajo (100 metros combinada II y 100 metros rescate con aletas) los aspirantes que realizan el test en vaso de 25 metros, pueden tocar y empujarse sobre la pared 2 veces más, que aquellos que realizan la prueba en vaso de 50 metros. Por lo tanto, realizar los test en vasos de 25 metros, permite que el aspirante disponga de más ocasiones para detener brevemente el nado, cada vez que llega al bordillo y además, recorrer los metros correspondientes a la fase de deslizamiento posterior a cada uno de los virajes, sin apenas consumir energía. Por todo esto, es comprensible que estas circunstancias generen sentimientos de inequidad entre los aspirantes a socorrista, ya que algunos aspirantes que son capaces de superar las pruebas en vaso de 25 metros, posiblemente no puedan superar estos mismos test en un vaso de 50 metros y viceversa.

En definitiva y como consecuencia de los resultados de este trabajo, se recomienda que si las instituciones que otorgan las certificaciones de socorrista consideran que este tipo de pruebas cronometradas debe mantenerse, es necesario que los baremos de tiempo de dichas pruebas, se adapten a la longitud del vaso en el que se evalúan. No obstante, dado el amplio margen de tiempo disponible para realizar las pruebas estudiadas en esta investigación, también 
sería deseable que los aspirantes socorrista dispusieran de un nivel de destreza en el agua lo suficientemente alto, como para ser capaces de superar holgadamente las pruebas.

\section{CONCLUSIONES}

Los resultados de este estudio demuestran que la longitud del vaso en el que se evalúa a los aspirantes a socorrista, influye sobre el porcentaje de aspirantes que superan las pruebas y sobre las marcas de tiempo que se acreditan para su realización.

\section{REFERENCIAS BIBLIOGRÁFICAS}

Abraldes, J. A., Fernandes, R. J., Soares, S., Lima.;, A. B., \& Vilas-Boas, J. P. (2010). Assessment of A Lifesaver's Instantaneous Velocity in Mannequin Carry using Diferent Types of Fins. The Open Sports Sciences Journal,, 3(19), 19-21. https://doi.org/10.2174/1875399X01003010019

Barcala-Furelos, R., Szpilman, D., Palacios-Aguilar, J., Costas-Veiga, J., Abelairas Gómez, C., Bores-Cerezal, A., . . Rodríguez-Núñez, A. (2016). Assessing the efficacy of rescue equipment in lifeguard resuscitation efforts for drowning. The American $\begin{array}{llll}\text { Journal of } & \text { Emergency }\end{array}$ https://doi.org/10.1016/i.ajem.2015.12.006 PMid:26782793

Consejería de Sanidad y Consumo. (2006). Orden 1319/2006, de 27 de junio, de la Consejería de Sanidad y Consumo, por la que se establecen los criterios que permitan establecer los niveles de formación del personal que preste sus servicios como socorrista en piscinas, instalaciones acuáticas y medio natural de la Comunidad de Madrid. Boletín Oficial de la Comunidad de Madrid 14 de julio,(166), 53-82.

Cruz Roja Española. (2017). Socorrista en piscinas e instalaciones acuáticas, 2017, from http://www.cruzroja.es/principal/documents/1151742/1524540/socorrista.pdf/dc366 3c4-f74f-429f-aa4f-8f3281bdf8b3

Ellis \& Associates. (2002). El profesional del rescate acuático. Barcelona: Paidotribo.

Federación Madrileña de Salvamento y Socorrismo. (2017). Curso de Formación de Socorrista en Playa y Medio Natural 2017, from http://www.fmss.es/ajax.php?accion=modalcomo-es-el-curso\&id_curso=374

García Sanz, A., García Sanz, J. L., \& Díez Herrero, J. M. (2015). Técnicas de rescate y lesión medular en el medio acuático (Real Federación Española de Salvamento y Socorrismo ed.). España: Difusión Avances de Enfermería.

International Life Saving Federation. (2000). International Life Saving Federation, Pool Lifeguard 2017, from http://www.ilsf.org/sites/ilsf.org/files/Certification/ILSCertificates/APP\%2008\%20ILS $\% 20$ Lifeguard\%20Pool.pdf

International Life Saving Federation. (2007). Fitness Testing for Lifeguards, from http://www.ilsf.org/content/fitness-testing-lifeguards

International Life Saving Federation. (2013). International Life Saving Federation, beach lifeguard., 2017 ,

from http://www.ilsf.org/sites/ilsf.org/files/Certification/ILSCertificates/APP\%2010\%20ILS \%20Lifeguard\%20Beach.pdf

Keskinen, K., Keskinen, O., \& Mero, A. (2007). Effect of pool length on blood lactate, heart rate, and velocity in swimming. International Journal of Sports Medicine, 28, 407413.https://doi.org/10.1055/s-2006-924505 PMid:17111309 
Koch-Ziegenbein, P., Knechtle, B., Rüst, C. A., Rosemann, T., \& Lepers, R. (2013). Differences in swimming speed on short course and long course for female and male breaststroke swimmers: A comparison of swimmers at national and international level. OA Sports Medicine https://doi.org/10.13172/2053-2040-1-2-910

Lowensteyn, I., Perry, A., Nash, M., \& Salhanich, D. (1994). Differences in peak blood lactate concentration in long course versus short course swimming. Journal of Swimming Research., 10, 31-34.

Ministerio de Trabajo e Inmigración. (2011). Real Decreto 711/2011, de 20 de mayo, por el que se establecen tres certificados de profesionalidad de la familia profesional Actividades físicas y deportivas que se incluyen en el Repertorio Nacional de certificados de profesionalidad. Boletín Oficial del Estado de 20 de mayo (711), 59404-59439.

Palacios-Aguilar, J., López-García, S., Navarro-Patón, R., \& L.; C.-R. (2012). Rescue material: The flippers. The chance of surviving of a victim of a cardiac arrest

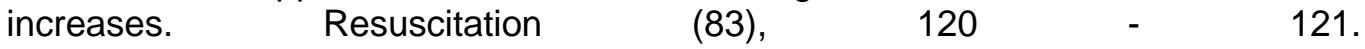
https://doi.org/10.1016/j.resuscitation.2012.08.310

Palacios Aguilar, J. (2008). Socorrismo acuático profesional "formación para la prevención y la intervención de accidentes en el medio acuático. A Coruña SADEGA.

Palacios Aguilar, J. (2010). IV Congreso Internacional de Salvamento Acuático, Rescate y Reanimación Cardiopulmonar "El Socorrismo en la actualidad, una actividad vital y cada vez más compleja"'. Paper presented at the El beneficio de la utilización de aletas en Socorrismo Acuático, POSADAS. Misiones. Argentina.

Palacios Aguilar, J. (2012). II CONGRESO INTERNACIONAL DE ACTIVIDADES ACUÁTICAS, SALVAMENTO Y SOCORRISMO. "La importancia de la capacitación en las actividades acuáticas, socorrismo y el salvamento". Paper presented at the Técnicas para incorporar en el salvamento: El beneficio en la utilización de aletas en socorrismo acuático POSADAS. Misiones. Argentina.

Real Federación Española Natación. (2016). Normativas natación, aspectos generales. Temporada 2016-2017, from http://www.rfen.es/publicacion/userfiles/NAT_00_Normativa_ASPECTOS_GENER ALES_2016-2017.pdf

Real Federación Española Salvamento y Socorrismo. (2003). Salvamento acuático y primeros auxilios. Madrid: Real Federación Española de Salvamento y Socorrismo.

Real Federación Española Salvamento Socorrismo. (2016). Mínimas individuales campeonato de España. Normativa de competición 2016/2017, from https://salvamentopalencia.files.wordpress.com/2013/03/minimas-invierno-2017.pdf

Sanz Arribas, I. (2011). La coordinación de socorristas en piscinas con gran superficie de lámina de agua. Revista Internacional de Medicina y Ciencias de la Actividad Física y el Deporte, 11(44), 650-673.

Sanz Arribas, I. (2015). Jornadas Técnicas de Salvamento y Socorrismo 2015. Paper presented at the El aro salvavidas versus el tubo de rescate en las instalaciones acuáticas, Madrid.

Sanz Arribas, I. (2016). Estudio comparativo del tiempo de intervención con aro salvavidas $y$ tubo de rescate. Paper presented at the $\mathrm{V}$ Congreso Internacional de Actividades Acuáticas. Celebrado los días 1, 2 y 3 de Julio de 2016 en la ciudad de Benidorm (Alicante) España., $\quad$ Benidorm PMid:27497397

Sanz Arribas, I., Aguado Gómez, R., \& Martínez de Haro, V. (2017). Influencia de las aletas sobre el tiempo de ejecución en los rescates de víctimas con parada cardiorespiratoria Retos. Nuevas tendencias en Educación Física, Deportes y Recreación(31), 133-136.

Schwebel, D. C., Heater, J., Holder, E., \& Marciani, F. (2010). Lifeguards: A forgotten aspect of drowning prevention. Injure \& Violence, 2(1), 1-3. https://doi.org/10.5249/jivr.v2i1.32 PMid:21483192 PMCid:PMC3134895 
The United States Lifesaving Association. (2016). Guidelines for open water lifeguard agency certificaction, http://c.ymcdn.com/sites/www.usla.org/resource/resmgr/docs/usla_certguidelines.p df

Wolfrum, M., Knechtle, B., Rüst, C. A., Rosemann, T., \& Lepers, R. (2013). The effects of course length on freestyle swimming speed in elite female and male swimmers - a comparison of swimmers at national and international level. SpringerPlus, 2, 643. https://doi.org/10.1186/2193-1801-2-643 PMid:24349949 PMCid:PMC3862862

Wolfrum, M., Rüst, C. A., Rosemann, T., Lepers, R., \& Knechtle, B. (2014). The Effect of Course Length on Individual Medley Swimming Performance in National and International Athletes. Journal of Human Kinetics, 42, 187-200. https://doi.org/10.2478/hukin-2014-0073

PMid:25414752 PMCid:PMC4234758

Número de citas totales / Total references: 33 (100\%)

Número de citas propias de la revista / Journal's own references: 1 (3.03\%)

Rev.int.med.cienc.act.fís.deporte - vol. 18 - número 72 - ISSN: 1577-0354 